\title{
THE STRUCTURE OF THE PROMINENCE-CORONA INTERFACE
}

\author{
F. Q. OR RALL \\ Institute for Astronomy, University of Hawaii, Honolulu, Hawaii, U.S.A. \\ and \\ R. J. SPEER \\ Dept. of Physics, Imperial College, London, England
}

\begin{abstract}
The relatively cool $\mathrm{H} \alpha$ emitting regions of solar prominences are evidently surrounded by an interface or structure of intermediate temperature that separates them from the surrounding hot corona. At present, very little is known about this prominence-corona interface (hereafter called the PC interface), because most of its distinctive radiation is in the EUV where observations of high spatial resolution have not been available. In this paper we report on studies made on photometrically calibrated monochromatic images of prominences and the surrounding corona recorded on slitless spectra made during the eclipse of 1970, March 7. These were obtained with a rocket-borne Wadsworth spectrograph flown by the consortium group (Speer et al., 1970) and cover the range $\lambda \lambda 850-2159$ with a spectral resolution of $0.17 \AA$ and a spatial resolution better than $10^{\prime \prime}$ over half the spectrum. They include spectral lines of a number of ionic species from $\mathrm{HI}$ through $\mathrm{Nixv}$. Because each species exists in a rather narrow temperature range, some of the monochromatic images show the low temperature prominence itself; some the coronal structure surrounding it; and some the intermediate structure we have called the PC interface.

At the time of the eclipse there were 10 prominences visible at the Sun's limb on $\mathrm{H} \alpha$ ciné observations made concurrent with the eclipse with the dual coronagraph at Mt. Halekala. Images of all ten prominences are visible on the EUV spectra in emission lines from a number of ionic species from $\mathrm{HI}$ through $\mathrm{OVI}\left(T \sim 3 \times 10^{5} \mathrm{~K}\right)$. Images in lines formed at $T \geqslant 3 \times 10^{5} \mathrm{~K}$ do not show the prominences, although they may show other coronal structures nearby. This is consistent with the finding of Noyes et al. (197.2) that the contrast of prominences against the coronal background drops sharply for lines formed with $T \geqslant 3 \times 10^{5} \mathrm{~K}$.

Two prominences of the 'coronal rain' type (hereafter called prominences $A$ and $B$, respectively) were especially well suited for a study of the PC interface. They have a simple geometry (apparently cylindrical) and the orientation of the magnetic field is known to be aligned parallel to the axis of symmetry of the cylinder.

Figure 1 shows the total corrected width at half intensity of these prominences as measured in several emission lines and plotted vs the temperature where the line is formed. These results suggest that these prominence threads consist of a cool $\mathrm{H} \alpha$ emitting core about $10^{9} \mathrm{~cm}$ in diameter surrounded by a thin sheath in which the temperature climbs steeply to at least $3 \times 10^{5} \mathrm{~K}$. At higher temperatures the width
\end{abstract}


becomes uncertain because the contrast becomes low or because other features become visible which become confused with the prominence structure.

We have determined the absolute total intensities of all of the measurable lines in prominences $A$ and $B$. The quantity measured for each line is the total intensity in the line from a slice of the prominence $1 \mathrm{~cm}$ thick, the slice being taken in the plane normal

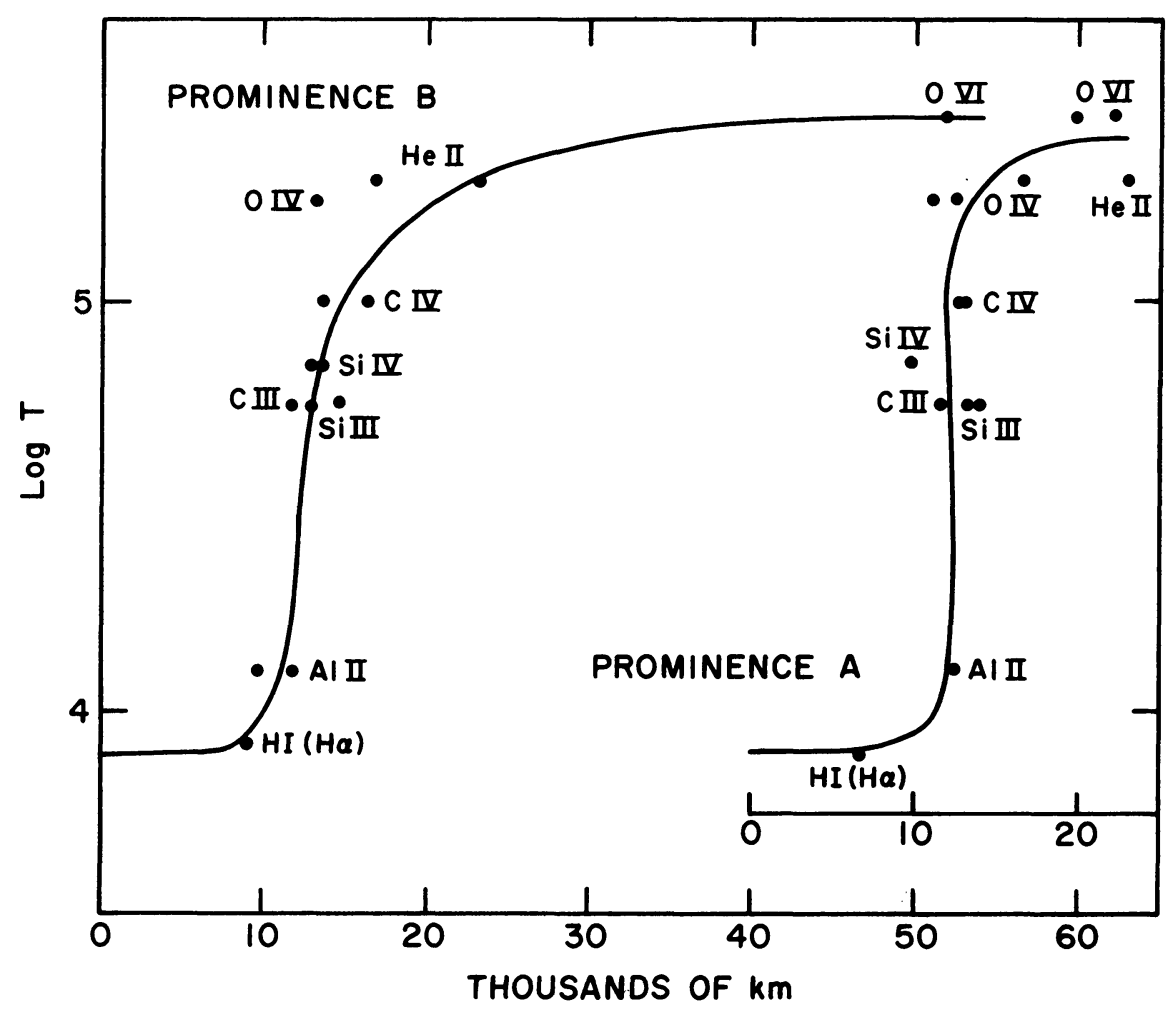

Fig. 1. The total corrected width at half-intensity measured in several emission lines, plotted against the logorithm of the temperature where the line is formed.

to the axis of symmetry of the thread. The techniques that have been developed to study the EUV emission of the chromosphere-corona transition region (hereafter called the $\mathrm{CC}$ transition) are also applicable to prominences with slight modification (see e.g., Athay, 1971; Noyes and Withbroe, 1973). In the analysis we have used the EUV coronal abundances and (where possible, the atomic parameters given by Dupree (1972) and the characteristic temperatures given by Burton et al. (1971).

Among the measured lines are the density sensitive pair $\lambda 1176$ and $\lambda 1909$ of $\mathrm{CIII}$. In prominence $B$, the intensity ratio of the CIII lines is observed to be $I(\lambda 1176) /$ $I(\lambda 1909)=1.45$. From the calculations of Gabriel and Jordan (1972) this ratio implies $N_{\mathrm{e}}=3 \times 10^{9} \mathrm{~cm}^{-3}$. Since this ion is formed at $T_{\mathrm{e}} \sim 56000 \mathrm{~K}$, the product $N_{\mathrm{e}} T_{\mathrm{e}} \simeq 1.7 \times$ $10^{14} \mathrm{~cm}^{-3} \mathrm{deg}$. We shall assume that $\phi \equiv N_{\mathrm{e}} T_{\mathrm{e}} \sim 2 \times 10^{14} \mathrm{~cm}^{-3} \mathrm{deg}$ in both prom- 
inences, corresponding to a total gas pressure of $\sim 0.06$ dyne within the PC interface. Analysis of the EUV resonance lines contains implicit information on the temperature gradient (Athay, 1966). Given the abundance, each measured line intensity yields the quantity

$$
\phi^{2}\left[\frac{1}{r} T^{5 / 2} \frac{\mathrm{d} T}{\mathrm{~d} r}\right]^{-1},
$$

where the bracketed quantity is appropriate to the temperature where the observed spectral line is formed. Here $r$ can be inferred from Figure 1. The results of applying this analysis to the measured intensity is shown in Figure 2 where we have plotted

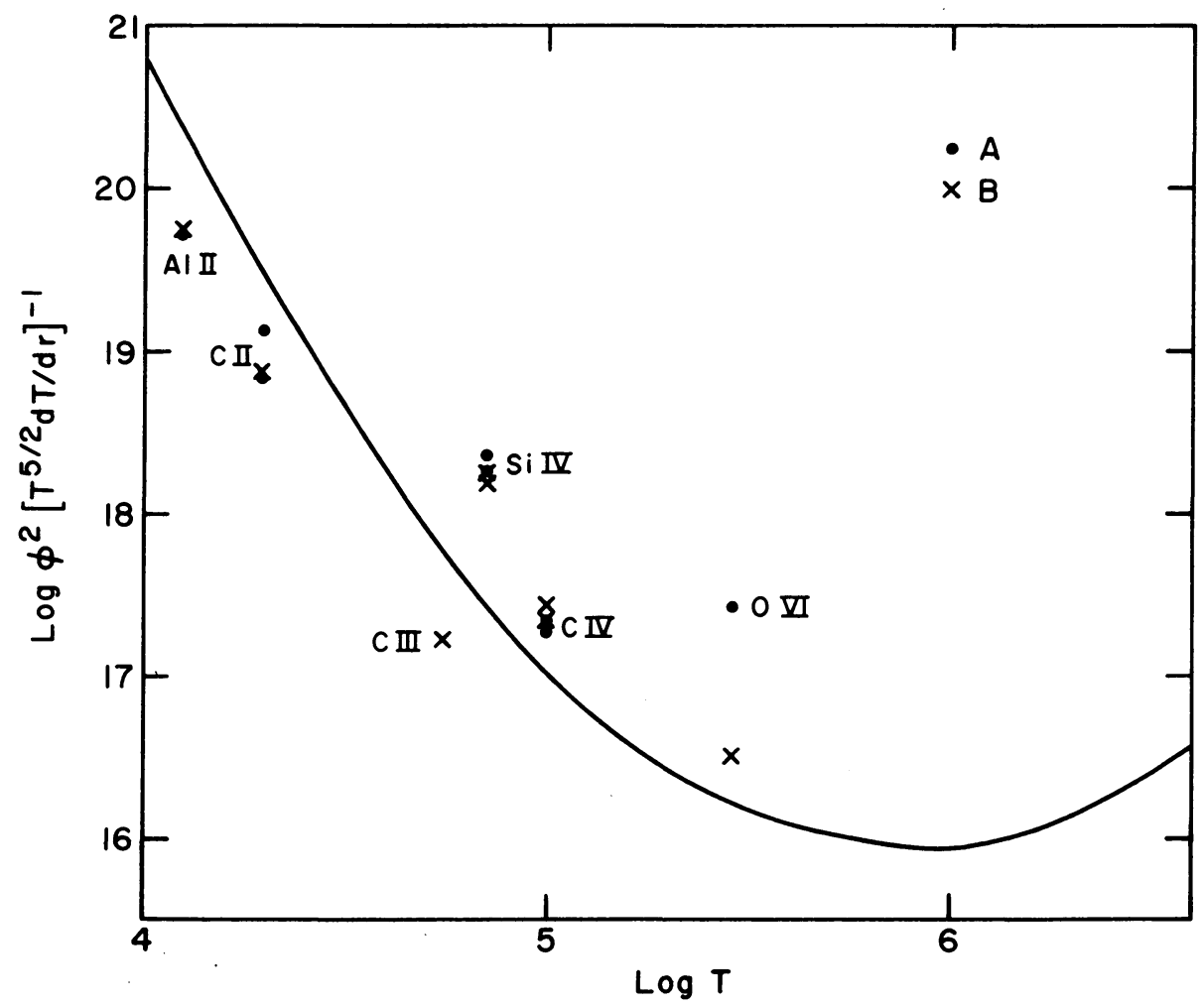

Fig. 2. The temperature dependence of $\log \phi^{2}\left[T^{5 / 2} \mathrm{~d} T / \mathrm{d} r\right]^{-1}$ derived from resonance line intensities. The plotted points are for prominences $A$ and $B$. The solid curve represents Kopp's (1972) results for the CC transition region, displaced downward to compensate for the lower pressure in prominences.

$\log \phi^{2}\left[T^{5 / 2} \mathrm{~d} T / \mathrm{d} r\right]^{-1}$ determined from each line vs the log of the temperature where that line is formed. The results from both prominences are plotted and are in good agreement, except for $\mathrm{O}$ vi. The solid line in Figure 2 represents the same quantity determined in the CC transition region by Kopp (1972) using a similar technique. We have displaced Kopp's curve downward to compensate for the fact that the pressure is a factor four higher in the transition region than in the prominence. 
We do not wish to overstress the similarity between the PC interface and the CC transition region suggested by Figure 2. Nevertheless, it is worthy of note that within the scatter of both sets of data, the results are identical in the range $T=4 \times 10^{5}$ and $10^{5} \mathrm{~K}$. Taken literally, this would imply that $\nabla T$ as a function of $T$ is identical in the lower transition region and in the PC interface of these transient prominences. This is surprising. We expect the temperature gradient to be sensitive to the orientation and magnitude of the magnetic field, which is quite different in the transition region and in these prominences. However, these prominences are changing in time and may not even approximate the steady state, and thus may not be typical. We are making a similar study of quiescent prominences on the same plates.

This work was supported in part under NASA Grant NGL 12-001-011.

\section{References}

Athay, R. G.: 1966, Astrophys. J. 145, 784.

Athay, R. G.: 1971, in C. J. Macris (ed.), Physics of the Solar Corona, D. Reidel Publ. Co., Dordrecht, p. 36. Burton, W. M., Jordan, C., Ridgeley, A., and Wilson, R.: 1971, Phil. Trans. Roy. Soc. Lord. A270, 81. Dupree, A.: 1972, Astrophys. J. 178, 527.

Gabriel, A. H. and Jordan, C.: 1972, Case Studies in Atomic Collision Physics, vol. 2, North-Holland Publ. Co., Amsterdam.

Kopp, R.: 1972, Solar Phys. 27, 373.

Noyes, R. W., Dupree, A. K., Huber, M. C. E., Parkinson, W. H., Reeves, E. M., and Withbroe, G. L. : 1972, Astrophys. J. 178, 515.

Noyes, R. W. and Withbroe, G. L.: 1972, Space Sci. Rev. 13, 612.

Speer, R. J., Garton, W. R. S., Goldberg, L., Parkinson, W. H., Reeves, E. M., Morgan, A. F., Nicholls, R. W., Jones, T. J. L., Paxton, J. H. B., Shenton, D. B., and Wilson, R.: 1970, Nature 226, 249.

\section{DISCUSSION}

Jordan: I have seen your preprint and couldn't resist the temptation to put some of my own numbers into your computations. Using my CIII population and ionization equilibrium calculations I get a pressure which is a factor of two higher than the one you get. Also using the observed Fe XII, intensities in the corona and my own calculations for the populations you get a ridiculously low electron density, $10^{7} \mathrm{~cm}^{-3}$. Therefore I now modify my own calculations by about a factor of 2 . This leads to electron densities in the quiet corona of about $2 \times 10^{8} \mathrm{~cm}^{-3}$, and it raises the electron density in the region you have studied up to $10^{9} \mathrm{~cm}^{-3}$. Thus the pressure in the corona surrounding the prominence is about a factor of 3 larger than in the prominence and you would need a magnetic field of about 6 Gauss in the prominence to maintain equilibrium. Further you have really not compared your $\int \mathrm{Ne}^{2} \mathrm{~d} H$ curve with the best available data. We have far more points at the low temperature and of the curve between $10^{4}-10^{5} \mathrm{~K}$ and this is much shallower than the curve that you showed from Duprea which was varying very rapidly. If you compared your results with our absolute intensities you would find that they agree much better and you will not have to scale your pressure so much.

Gabriel: The suggestion that the transition region should be thin because the magnetic field is parallel to the prominence is perhaps not valid because it is not a steady state phenomenon. As you pointed out the prominence is a falling or condensing coronal ring. It could have an intermediate temperature region surrounding it which is dragged on the magnetic field lines and has nothing to do with conduction from the corona to the cooler prominence material. 